Original

\title{
Nefrectomía radical laparoscopica. Técnica, resultados y complicaciones
}

\author{
Alfredo Aguilera Bazán, Manuel Pérez Utrilla, Manuel Girón, Jesús Cisneros Ledo, \\ Javier de la Peña Barthel
}

Hospital Universitario La Paz. Madrid, España

\begin{abstract}
Resumen
El tratamiento de elección del carcinoma renal de células claras es un tratamiento quirúrgico, siendo un tumor que no responde a quimioterapia, radioterapia o inmunoterapia. Dicho tratamiento quirúrgico ha ido variando a lo largo del tiempo en los últimos 40 años, debido sobre todo al desarrollo y universalización de los sistemas de diagnóstico como la ecografía y la TAC. Como consecuencia directa, la cirugía conservadora de parénquima deja de ser empleada solamente en pacientes monorrenos y comienza a extenderse sus indicaciones en pacientes con tumores de hasta $4 \mathrm{~cm}$ de diámetro, demostrando ser una alternativa segura y eficaz a la nefrectomía radical.

El otro hito importante en el desarrollo del tratamiento quirúrgico del cáncer renal es sin duda la revolución iniciada en la década de los 90 con el inicio de la cirugía renal laparoscópica, que ha venido implantándose en los últimos 20 años aproximadamente. En unos comienzos dubitativos en lo que a seguridad oncológica se refiere, actualmente contamos con series lo suficientemente extensas en tiempo de seguimiento y tamaño muestral, como para pensar que se trata de una técnica lo suficientemente fiable. El desarrollo de la tecnología ha ayudado a que la técnica laparoscópica ya no se vea como una técnica de futuro, sino de absoluta actualidad que debería estar implantada en la mayoría de los servicios de Urología.

Toda implantación de una nueva técnica suele acarrear una serie de complicaciones que debemos estar dispuestos a asumir y a intentar poner todos los medios a nuestro alcance para evitarlas. Ante esta situación, es fundamental desarrollar un programa de formación y de inicio en la técnica llevado a cabo con sentido común, en el cual la selección de los pacientes y de las patologías a abordar es primordial.
\end{abstract}

Palabras clave: Cáncer renal. Nefrectomía laparoscópica. Complicaciones.

\section{Laparoscopic radical nephrectomy. Procedure, results, and complications}

\section{Abstract}

Surgery is the treatment of choice for clear cell renal carcinoma not responding to chemotherapy, radiotherapy, or immunotherapy. The surgical procedure used has evolved over time in the past 40 years, mainly because of the development and widespread use of diagnostic procedures such as ultrasonography and CT. As a direct consequence, parenchymal-sparing surgery was no longer only used for patients with solitary kidneys and its indication started to be extended to patients with tumors up to $4 \mathrm{~cm}$ in diameter, in whom it has been shown to be a safe and effective alternative to radical nephrectomy.

The other important milestone in development of renal cancer surgery undoubtedly was the revolution started in the 90s with advent of laparoscopic renal surgery, which has become established over the past 20 years approximately. Laparoscopic surgery initially raised concern about oncological safety, but clinical series with sufficiently long follow-up times and large sample sizes are now available to consider the laparoscopic approach as a reliable procedure. Technological development has caused the laparoscopic technique to be no longer seen as a procedure for the future, but rather as an absolutely current technique which should be implemented in most urology departments.

Implementation of any new procedure usually involves a number of complications which we should be prepared to assume, while making every effort to try and prevent them. It is therefore essential to develop, and to implement using common sense, a training program on the procedure. Selection of patients and conditions to be treated is a crucial part of such a program.

Keywords: Renal cancer. Laparoscopic nephrectomy. Complications.

$\mathrm{D}$ esde que en 1968 Robson et al. describieran la técnica de la nefrectomía radical clásica, con extirpación del riñón con la glándula suprarrenal incluida en la fascia de Gerota y una disección ganglionar ampliada, dicha cirugía ha sido universalmente aceptada como tratamiento de elección del carcinoma renal ${ }^{1}$.
Pero esta técnica ha ido experimentando cambios a lo largo del tiempo, así nos encontramos con que la suprarrenalectomía no se considera necesaria en todos los casos de tumor renal debido a la baja frecuencia en que la glándula se encuentra afectada. El porcentaje varía según los artículos, pero podría estar infiltrada en torno al $2 \%$ en las 
series modernas ${ }^{2,3}$, quedando limitada la suprarrenalectomía a aquellos tumores de polo superior o con sospecha de afectación radiológica. En estos estudios, parece que el tamaño de la masa y el estadio tumoral están correlacionados con la probabilidad de la afectación de la glándula.

Por otra parte, el papel de la linfadenectomía de carácter curativo se encuentra cada vez más debatido, siendo indicada en aquellos casos en que los ganglios sean positivos en estudios de imagen o durante la cirugía. De interés es la lectura del artículo de Pantuck et al en el que se revisan 900 linfadenectomías realizadas en el contexto de una nefrectomía radical por carcinoma renal, llegando a la conclusión previamente expuesta ${ }^{4}$.

A estos cambios hay que añadir la universalización de los métodos diagnósticos de imagen como la ecografía abdominal y la TAC durante la década de los 90, lo que hizo que un tumor de diagnóstico tardío y con una manifestación clínica por lo general inespecífica (triada clásica entre un 4-11\%) se comenzara a diagnosticar de manera incidental, con un menor tamaño y en un estadio precoz. El porcentaje actual de carcinoma renal que se diagnostica como incidentaloma es alto. Las cifras varían según los autores, por ejemplo, Jayson et al. lo cifran en un $61 \%$ en 1998 frente a un $13 \%$ en $1978^{5}$

Ante esta situación, la comunidad urológica se empezó a plantear la posibilidad de realizar cirugía renal conservadora (tumorectomía, heminefrectomía) de esos pequeños tumores en aquellos casos seleccionados de pacientes que quedarían anéfricos si se les realizara una nefrectomía radical. Posteriormente esta indicación única iría ampliándose a pacientes con el riñón contralateral normal. Varios son los trabajos que han estudiado la seguridad oncológica de la nefrectomía parcial, como ejemplo de ello nos encontramos con la exhaustiva revisión de la literatura desde 1980 hasta 2000 que hacen Uzzo y Novick, en el que se recogen los resultados de las series más importantes hasta el momento, alguna con un seguimiento de los casos de más de 10 años, como es el caso de Fergany et al. En dicha revisión, se llega a la conclusión de la seguridad de la técnica, con una tasa de recidiva para tumores de menos de $4 \mathrm{~cm}$ de entre 0 y $3 \%$. Este artículo describe la técnica en los detalles más importantes y analiza las diferentes indicaciones asî como la variabilidad de resultados obtenidos según el tamaño tumoral extirpado ${ }^{6}$. Algo más reciente es el estudio multicéntrico realizado por el Departamento de California y publicado en 2004 en el que se analiza la seguridad de la nefrectomía parcial frente a la nefrectomía radical en tumores T1. Tras analizar 1.454 pacientes (379 parciales, 1.075 radicales), llegan a la conclusión de que no existen diferencias significativas en cuanto a recidiva local o metástasis a distancia así como en muerte cáncer específica.

A esta evolución de la técnica hay que añadir la irrupción de la laparoscopia renal también en la década de los 90. Considerada por muchos autores como técnica de elección, con resultados oncológicos superponibles a la cirugía abierta en casos de tumores localizados ${ }^{8,9}$ también ha seguido la evolución de la nefrectomía radical hacia la cirugía conservadora laparoscópica, apareciendo la primera referencia en patología benigna en $1993^{10}$.

\section{TÉCNICA}

El abordaje laparoscópico para realizar una nefrectomía radical puede ser transperitoneal o accediendo directamente al retroperitoneo (lumboscopia). El más extendido de los dos es el transperitoneal, debido a sus conocidas ventajas en cuanto a mayor espacio de trabajo y mejor orientación en el campo quirúrgico ${ }^{11,12}$. La lumboscopia permite trabajar directamente en el retroperitoneo; la cavidad peritoneal permanece intacta, lo que se traduce en una menor distensión abdominal en el postoperatorio, menor riesgo de lesión intestinal y de órganos intraabdominales. Una tercera posibilidad es el acceso transperitoneal asistido por la mano. En este caso, la principal ventaja la encontramos al inicio del aprendizaje, ya que la mano nos sirve de referencia en la pérdida de la tercera dimensión por una parte y en la mayor seguridad que nos proporciona el hecho de tener la mano en el campo operatorio en caso de tener un accidente vascular, que sin duda es la principal preocupación en la cirugía retroperitoneal. Como desventaja, creemos que la disección es peor, ya que tenemos una mano ocupada permanentemente, sin poder emplearla para la disección más fina.

La generación del neumoperitoneo puede realizarse bajo visión directa con una pequeña incisión (aproximadamente 1-2 cm) o "a ciegas" con la aguja de Veress. Esta última permite un acceso más rápido, pero al ser una técnica cerrada debe ser utilizada por cirujanos con experiencia. En caso de pacientes 
con antecedentes quirúrgicos abdominales, debemos extremar las precauciones en cualquiera de los dos casos, debido al riesgo de lesión intestinal por adherencias.

Una vez generado el neumoperitoneo y colocados los puertos de trabajo (existen infinidad de combinaciones, validas según la experiencia de cada cirujano), es fundamental la orientación anatómica. En este sentido, la identificación del músculo psoas y del uréter y vena gonadal parece la vía más segura para desarrollar el plano que nos llevará al hilio renal, variando si es derecho o izquierdo las peculiaridades anatómicas que siempre debemos tener en mente (presencia de venas lumbares, proximidad de los grandes vasos, venas suprarrenales, etc...).

Tras la cuidadosa disección del hilio renal y sección de los vasos (parece que las suturas tipo endoGia pierden terreno frente a los clips, debido a la simplicidad de manejo de estos últimos), movilizamos el polo superior y la convexidad renal. Este dato es muy importante y hay que tenerlo en cuenta; hasta este momento no hemos movilizado la convexidad de la pieza, ya que de lo contrario estaría permanentemente descolgándose y dificultando la disección del hilio renal.

Una vez retirada la pieza, es importante revisar la hemostasia del lecho quirúrgico, para lo cual reduciremos la presión intraabdominal durante unos minutos, lo que permitirá visualizar el sangrado venoso que podría estar colapsado por la presión. También debemos revisar la retirada de los trócares para asegurarnos de que no se produce sangrado en este punto. La extracción de la pieza la realizamos con bolsa.

\section{RESULTADOS}

Aplicada inicialmente en el campo de la Ginecología y la Cirugía General, la laparoscopia tarda más tiempo en instaurarse en Urología debido sobre todo a que no existe una técnica frecuente y sencilla, como es el caso de la colecistectomía o la ligadura de trompas, con la que iniciarse y adquirir experiencia.

Uno de los argumentos esgrimidos inicialmente en contra de la laparoscopia como técnica de exéresis quirúrgica para casos oncológicos, se debe a la aparición de las primeras metástasis en puertos o diseminación peritoneal. Desde el primer caso descrito de metástasis en puertos en 1978 por una laparoscopia diagnóstica en un tumor de ovario, se han descrito más casos en la literatura, apareciendo la primera referencia urológica en 1994 por una linfadenectomía de un carcinoma vesical de alto grado en la cual no se empleó bolsa de extracción ${ }^{13}$. En una revisión de 10.912 procedimientos laparoscópicos urológicos por cáncer, se registraron 13 casos de implantes tumorales $(0,1 \%)$, en 10 de ellos se localizaron en los puertos $(0,09 \%)$ y en 3 casos en peritoneo $(0,03 \%)$. De estos 13 casos, 4 fueron en nefrectomías radicales en los que se morceló la pieza dentro de la bolsa de extracción en todos ello $^{14}$. En otra revisión sobre 1.098 intervenciones urológicas, se detectaron 8 recurrencias en puertos, de las cuales 1 correspondía a una nefrectomía radical $^{15}$. Existen más casos descritos en la literatura de implantes tumorales en los puertos de trabajo, sobre todo en intervenciones de Cirugía General y Ginecología ${ }^{16,17}$.

No está claro el mecanismo responsable de la metástasis en puertos, aunque todo parece indicar que se debe tratar de un proceso multifactorial en el cual el grado de experiencia del cirujano juega un papel importante. Otros factores relacionados con este proceso podrían ser el grado tumoral, rotura del tumor, contaminación del instrumental, neumoperitoneo, efecto chimenea de los trócares, etc. Muy recomendable es la lectura del artículo de Tsivian et al. en el que se analizan todos estos factores y todos los casos urológicos descritos hasta el momento ${ }^{18}$. Diferentes estudios multicéntricos de Cirugía General parecen apoyar cada vez más la idea de que la técnica laparoscópica (generación de neumoperitoneo, colocación de trócares) es oncológicamente segura ${ }^{19-21}$.

En lo que se refiere al caso concreto del tratamiento quirúrgico del cáncer renal mediante laparoscopia, técnicamente es una cirugía reproducible que parece cumplir los criterios oncológicos de la técnica abierta, pudiéndose extraer el riñón con la grasa perirrenal, fascia de Gerota y si es necesario la glándula adrenal o linfadenectomía, con un control precoz del hilio renal.

El hecho de que técnicamente la cirugía se pueda realizar y sea reproducible, no quiere decir que el control oncológico sea bueno y se deba realizar. Para llegar a esta conclusión es necesario tener resultados a largo plazo de series de gran tamaño muestral $^{13,22}$. En el caso del cáncer renal estos resultados ya se han obtenido y la conclusión a la que se llega es que la nefrectomía radical laparoscó- 
pica en tumores renales T1-2 es un estándar de tratamiento admitido, con una supervivencia libre de enfermedad comparable a la cirugía abierta, con menor morbilidad (nivel de evidencia 3) y que debería ser realizada en centros con experiencia laparoscópica (grado B de recomendación) ${ }^{23}$. En lo que se refiere al tamaño máximo tumoral que puede ser abordado por laparoscopia, diferentes autores ponen el límite en $15 \mathrm{~cm}^{24}$. Técnicamente complejo, debido al menor espacio de trabajo, mayor cantidad de neovascularización con un mayor riesgo de sangrado, más difícil movilización de la pieza, sin embargo, son casos que se pueden abordar a medida que se adquiere experiencia en laparoscopia. Son varios los artículos en la literatura que describen series de tumores con un tamaño por encima de los $9 \mathrm{~cm}$. En el estudio de Dillenburg et al. $^{25}$, en un total de 23 nefrectomías de tumores con un tamaño medio de $8,9 \mathrm{~cm}$, llegan a la conclusión de unos mejores resultados en los casos laparoscópicos frente a los abiertos en lo que se refiere a índices de calidad de vida y $100 \%$ de márgenes tumorales negativos. Steinberg et al. ${ }^{26}$ describen su serie de 62 nefrectomías laparoscópicas de tumores en estadio T2 con una media de tamaño tumoral de $9,2 \mathrm{~cm}$, comparándola con 32 casos abiertos, con unos resultados de menor tiempo quirúrgico, pérdida de sangre y estancia postoperatoria para los casos laparoscópicos.

Las guías de la Asociación Europea de Urología también plantean como posible el control oncológico en tumores T3a. En este sentido y todavía sin el seguimiento oncológico y tamaño muestral necesario, encontramos en la literatura artículos en los que se describen el abordaje laparoscópico de tumores renales con trombo en cava. Un ejemplo de esto es el artículo de Desai y Gill del año $2003^{27}$ en el que se describe una serie de 16 casos con trombos tumorales con afectación de la vena renal (nivel I de afectación) en los que se realizó el clampaje de la vena renal con una sutura mecánica laparoscópica ceñida al ostium de la vena. En todos los casos se exprimió el trombo hacia el riñón y no fue necesaria la apertura de la vena cava. En este artículo se describe también un modelo animal para realizar esta intervención con afectación de la cava por el trombo tumoral. Existen otras publicaciones en las que se describen la realización mano asistida de una nefrectomía radical derecha con trombo en cava. En los artículos de Sundaram et al. ${ }^{28}$ y Varkarakis et al. ${ }^{29}$ se presentan casos de pacientes con trombo tumoral de $1 \mathrm{~cm}$ y $2 \mathrm{~cm}$ de longitud en la vena cava respectivamente y un abordaje asistido por la mano transperitoneal en ambos. Si bien en el primero toda la nefrectomía fue realizada con la mano dentro del abdomen y previamente se realizó una embolización de la arteria renal, en los 4 casos descritos por Varkarakis la disección de la vena renal y de la cava fue enteramente laparoscópica y previamente no se embolizó la arteria. En un artículo del año 2005 de Disanto et al. ${ }^{30}$ se presenta un caso una mujer de 87 años con un tumor renal derecho y trombo tumoral de $7 \mathrm{~cm}$ de longitud que se resuelve asistido por la mano por via retroperitoneoscópica. A medida que adquirimos experiencia y se desarrolla el instrumental laparoscópico adecuado, nos planteamos la posibilidad de realizar estos casos técnicamente complejos y que precisan de un mayor seguimiento para conocer su seguridad oncológica.

Otro reto que nos plantea la laparoscopia renal es la posibilidad de realizar cirugía citorreductora en la enfermedad metastásica. El hecho de plantearnos estos casos por laparoscopia no debe tomarse como un alarde quirúrgico, sino como una ventaja para el paciente ya que al reducirse el periodo de recuperación postoperatorio, permite iniciarse el tratamiento complementario antes que si la nefrectomía se hace por vía abierta. Si bien es cierto, estos casos suelen ser técnicamente más difíciles ya que el tamaño tumoral normalmente es mayor, suele haber una mayor presencia de adenopatias perihiliares lo que dificulta la disección, invasión local o una mayor neovascularización, lo que se traduce en un porcentaje mayor de reconversiones a cirugía abierta. Un estudio comparativo entre nefrectomía laparoscópica citorreductiva y cirugía abierta demostró una estancia hospitalaria más corta $(2,3$ vs 6,1 días), menor sangrado intraoperatorio (288 vs $1.288 \mathrm{ml}$ ) y un inicio más precoz en la terapia sistémica (36 vs 61 días) en la laparoscopia ${ }^{31}$.

\section{COMPLICACIONES}

En la literatura encontramos referencias de estudios que analizan las complicaciones de procedimientos urológicos laparoscópicos. Los resultados de estos análisis varían con el tiempo, ya que se adquiere experiencia en la técnica y por otra parte cada vez se realizan casos más complejos. Así, en 1996, Gomella et al. ${ }^{32}$ publican una tasa de complicaciones del 7,98\% para cirugía urológica como la nefrectomía radical, linfadenectomía pélvica o adre- 
nalectomía. Ese mismo año, un estudio multicéntrico alemán ${ }^{33}$ con 2.407 procedimientos urológicos describe un $4,4 \%$ de complicaciones.

En estudios más recientes publicados en $2007^{34}$, encontramos una publicación con 2.775 cirugías urológicas, de las cuales 549 son nefrectomías radicales (19,7\%). Analizado por procedimiento, describen como la complicación más frecuente en la nefrectomía radical laparoscópica la lesión de órgano adyacente en un $2,7 \%$ de los casos, la lesión vascular en un 2,2\%, el sangrado postoperatorio con necesidad de transfusión en un 1,3\% y el ileo paralítico en un 1,3\%. El porcentaje de reconversión a cirugía abierta es de un 2,9\%. $\mathrm{El}$ porcentaje de complicaciones mayores es del 7,3\%. La mortalidad total del estudio es del 0,07\%.

En un meta-análisis publicado en $2006^{35}$ donde se analizan 56 referencias en MEDLINE sobre complicaciones en cirugía renal, presentan el sangrado venoso como la principal complicación en la nefrectomía radical $(1,8 \%)$ de un total de 1.746 pacientes. La tasa global de complicaciones es del 10\%. En este estudio también se analizan las nefrectomías radicales asistidas por la mano, presentando como única diferencia un mayor porcentaje de infección de herida quirúrgica (1,5\% vs $0,2 \%)$.

Encontramos una gran disparidad de complicaciones descritas en la literatura relacionadas con la cirugía renal laparoscópica; lesiones de páncreas, transección de vena cava, apertura de diafragma, embolia gaseosa, etc... Por lo general, suelen tener una relación directa con la experiencia del cirujano y una buena selección de casos. De hecho, estas revisiones sobre complicaciones suelen distinguir entre un primer periodo de aprendizaje de la técnica y una segunda fase donde la tasa de complicaciones disminuye considerablemente. En este sentido, un reciente artículo de Kavoussi et al. analiza la tasa de reconversión a cirugía abierta de un total de 2.128 procedimientos laparoscópicos retroperitoneales, con una tasa global de 3,2\% (68 casos), que es de un $8 \%$ en 1999 y de un $1,3 \%$ en 2005. La principal causa de reconversión es la lesión vascular $(36,8 \%)$. La cirugía más frecuentemente reconvertida es la nefroureterectomía $(8,4 \%)$, necesitando reconvertir la nefrectomía radical a cirugía abierta en un 2,9\% de los casos. En todo caso, se trata de un tipo de cirugía en el que el campo quirúrgico donde trabajamos es el de los grandes vasos y cualquier error, normalmente por desorientación, puede tener consecuencias fatales.

\section{REFERENCIAS}

1. Robson CJ, Churchill BM, Anderson W.The results of radical nephrectomy for renal cell carcinoma. J Urol.1969 Mar;101(3): 297-301

2. Tsui KH, Shvarts O, Barbaric Z, Figlin R, de Kernion JB, Belldegrun A. Is adrenalectomy a necessary component of radical nephrectomy? UCLA experience with 511 radical nephrectomies. J Urol. 2000;163(2):437-441.

3. Kletscher BA, Qian J, Bostwick DG, et al: Prospective analysis of the incidence of ipsilateral adrenal metastasis inlocalized renal cell carcinoma. J Urol 1996;155(6):1844-1846.

4. Pantuck AJ, Zisman A, Dorey F, Chao DH, Han KR, Said J, Gitlitz BJ, Figlin RA, Belldegrun AS. Renal cell carcinoma with retroperitoneal lymph nodes. Role of lymph node dissection.J Urol. 2003;169(6):2076-2083.

5. Jayson M, Sanders H: Increased incidence of serendipitously discovered renal cell carcinoma. Urology. 1998;51(2):203-205.

6. Uzzo RG, Novick AC. Nephron sparing surgery for renal tumors: indications, techniques and outcomes. J Urol 2001;166(1):6-18.

7. Patard JJ, Shvarts O, Lam JS, Pantuck AJ, Kim HL, Ficarra V, Cindolo L, Han KR, De La Taille A, Tostain J, Artibani W, Abbou CC, Lobel B, Chopin DK, Figlin RA, Mulders PF, Belldegrun AS. Safety and efficacy of partial nephrectomy for all T1 tumors based on an international multicenter experience. J Urol. 2004;171(6 Pt 1):2181-2185.

8. Makhoul B, De La Taille A, Vordos D, Salomon L, Sebe P, Audet JF, Ruiz L, Hoznek A, Antiphon P, Cicco A, Yiou R, Chopin D, Abbou CC.. Laparoscopic radical nephrectomy for $\mathrm{T} 1$ renal cancer: the gold standard? A comparison of laparoscopic vs open nephrectomy. BJU Int. 2004 Jan;93(1):67-70.

9. Portis AJ, Elnady M, Clayman RV. Laparoscopic radical/tota nephrectomy. a decade of progress. J Endourol. 2001;15(4):345354.

10. Winfield HN, Donovan JF, Godet AS, Clayman RV. Laparoscopic partial nephrectomy: initial case report for benign disease. J Endourol. 1993;7:521-526.

11. Desai MM, Strzempkowski B, Matin SF, Steinberg AP, Ng C, Meraney AM, Kaouk JH, Gill IS. Prospective randomized comparison of transperitoneal versus retroperitoneal laparoscopic radical nephretctomy. J Urol. 2005;173(1):38-34.

12. Abdelmaksoud A, Biyani CS, Bagheri F, Janetschek G. Laparoscopic approaches in urology. BJU Int. 2005;95(2):244-249.

13. Ono Y, Hattori R, Gotoh M, Yoshino Y, Yoshikawa Y, Kamihira O. Laparoscopic radical nephrectomy for renal cell carcinoma: the standard of care already? Curr Opin Urol. 2005;15(2): 7578.

14. Micali S, Celia A, Bove P, De Stefani S, Sighinolfi MC, Kavoussi LR, Bianchi G. Tumor seeding in urological laparoscopy: an international survey. J.Urol 2004;171(6 Pt 1):2151-2154.

15. Rassweiler J, Tsivian A, Kumar AV, Lymberakis C, Schulze M, Seeman O, Frede T. Oncological safety of laparoscopic surgery for urological malignancy: experience with more than 1.000 operations. J Urol. 2003;169(6):2072-2075..

16. Cirocco WC, Schwartzman A, Golub RW. Abdominal wall recurrence after laparoscopic colectomy for colon cancer. Surgery 1994;116(5):842-846.

17. Jacquet P, Averbach AM, Jacquet N. Abdominal wall metastasis and peritoneal carcinomatosis after laparoscopic-assisted colectomy for colon cancer. Eur J Surg Oncol 1995;21(5):568570 .

18. Tsivian A, Sidi AA. Port site metastases in urological laparoscopic surgery. J Urol. 2003 Apr;169(4):1213-1238.

19. Schaeff B., Paolucchi, V. and Thomopoulos, J. Port site recurrences after laparoscopic surgery. A review. Dig Surg. 1998; 15(2): 124-134. 
20. Schiedeck TH, Schwandner O, Baca, I Baehrlehner E, Konradt J, Köckerling F, Kuthe A, Buerk C, Herold A, Bruch HP. Laparoscopic surgery for the cure of colorectal cancer: results of a German five-center study. Dis Colon Rectum. 2000; 43(1):1-8.

21. Zamora O, Gervaz P, Wexner S D. Trocar site recurrence in laparoscopic surgery for colorectal cancer. Surg Endosc. 2001; 15(8):788-793.

22. Dunn MD, Portis AJ, Shalhav AL, Elbahnasy AM, Heidorn C, McDougall EM, Clayman RV. Laparoscopic versus open radical nephrectomy:A nine year experience. J Urol 2000;164(4):11531159 .

23. Ljungberg B, Hanbury DC, Kuczyk MA, Merseburger AS, Mulders P, Patard JJ et al. Renal cell carcinoma guideline. European Urology 2007;51:1502-1510. (Traducción del artículo en Actas Urol Esp. 2009;33(3):270-279).

24. Malaeb BS, Sherwood JB, Taylor GDS, et al. Hand assisted laparoscopic nephrectomy for renal masses $>9,5 \mathrm{~cm}$ : series comparison with open radical nephrectomy. Urol Oncol. 2005; 23(5);323-327.

25. Dillenburg W, Poulakis V, Skriapas K, de Vries R, Ferakis N, Witzsch U, Melekos M, Becht E. Retroperitonoscopic vs open surgical radical nephrectomy for large renal cell carcinoma in clinic stage cT2 or cT3a: quality of life, pain and reconvalescence. Eur Urol. 2006;49(2):314-322.

26. Steinberg AP, Finelli A, Desai MM, Abreu SC, Ramani AP, Spaliviero M, Rybicki L, Kaouk J, Novick AC, Gill IS. Laparoscopic radical nephrectomy for large renal tumors. J Urol 2004;172(6 Pt 1): 2172-2176.

27. Desai MM, Gill IS, Ramani AP, et al: Laparoscopic radical neph rectomy for cancer with level I renal vein involvement. J Urol 2003;169(2):487-491.

28. Sundaram CP, Rehman J, Lamdman J, Oh J. Hand assisted laparoscopic radical nephrectomy for renal cell carcinoma with inferior vena caval thrombus. J Urol. 2002;168(1):176-179.
29. Varkarakis IM, Bhayanai S, Allaf M, Inagaki T, Gonzalgo ML, Jarrett TW. Laparoscopic-assisted nephrectomy with inferior vena cava tumor thrombectomy: preliminary results. Urology. 2004;64(5):925-929.

30. Disanto V, Pansadoro V, Portoghese F, Scalese GA, Romano M. Retroperitoneal Laparoscopic Radical Nephrectomy for Renal Cell Carcinoma with Infrahepatic Vena Caval Thrombus Eur Urol.2005; 47(3):352-356.

31. Rabets JC, Kaouk J, Fergany A, Finelli A, Gill IS, Novick AC. Laparoscopic vs open cytoreductive nephrectomy for metastasic renal cell carcinoma. Urology 2004;64(5):930-934.

32. Gomella LG, Abdel-Meguid TA, Lotfi MA, Hirsch IH, Albala D, Manyak M, Kozminski M, Sosa E, Stone NN. Laparoscopic urologic surgery outcome assessment. J Laparoendosc Adv Surg Tech A. 1997;7(2):77-86.

33. Fahlenkamp D, Rassweiler J, Fornara P,et al: Complications of laparoscopic procedures in urology:experience with 2,407 procedures at 4 German centers. J Urol 1999;162(3 Pt 1):765-770.

34. Permpongkosol S, Link RE, Su LM, Romero FR, Bagga HS, Pavlovich CP, Jarrett TW, Kavoussi LR..Complications of 2,775 Urological Laparoscopic Procedures: 1993 to 2005. J Urol. 2007;177(2):580-585.

35. Pareek G, Hedican S, Gee J et al. Meta-analysis of the Complications of Laparoscopic Renal Surgery: Comparison of Procedures and Techniques. J Urol. 2006;175(4):1208-1213.

Correspondencia autor: Dr. Alfredo Aguilera Bazán Servicio de Urología. Hospital Universitario La Paz. Madrid $\mathrm{P}^{\circ}$ de la Castellana, 261 - 28046 Madrid Tel.: 917277000

E-mail autor: aaguilera11@yahoo.es Información artículo: Original

Trabajo recibido: abril 2009

Trabajo aceptado: mayo 2009 